\title{
Dental Biometrics: Alignment and Matching of Dental Radiographs*
}

\author{
Hong Chen and Anil K. Jain \\ Department of Computer Science and Engineering \\ Michigan State University \\ East Lansing, MI 48824
}

\begin{abstract}
Dental biometrics utilizes the evidence revealed by dental radiographs for human identification. This evidence includes the tooth contours, the relative positions of neighboring teeth, and the shapes of the dental work (e.g., crowns, fillings and bridges). The proposed system has two main stages: feature extraction, and matching. The feature extraction stage uses anisotropic diffusion to enhance the images and a Mixture of Gaussians model to segment the dental work. The matching stage has three sequential steps: shape registration, computation of image similarity, and subject identification. In shape registration, we align the tooth contours and obtain the distance between them. A second method based on overlapped areas is used to match the dental work. The distance between the shapes of the teeth and the distance between the shapes of the dental work are then combined using likelihood estimates to improve the retrieval accuracy. At the second step, the correspondence of teeth between two given images is established. A distance measure based on this correspondence is then used to represent the similarity between the two images. Finally, the distances are used to infer the subject's identity.
\end{abstract}

\section{Introduction}

Dental biometrics automatically analyzes dental radiographs to achieve the aim of forensic dentistry, which is to identify the deceased individuals based on their dental characteristics. Based on the time of acquisition, there are two classes of dental radiographs. The radiographs acquired after the death are called the Post-mortem (PM) radiographs, and the radiographs acquired while the person is alive are called the Ante-mortem (AM) radiographs (Fig. 1). The AM radiographs are labelled with patients' names. The goal of dental biometrics is to match the unlabelled PM radiographs against the database of labelled AM radiographs. If the set of teeth in the PM radiographs sufficiently matches

* This research was supported by the National Science Foundation grant EIA-0131079. the teeth in an AM radiograph, the identity of the PM radiograph is obtained.

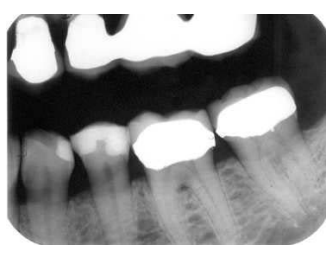

(a)

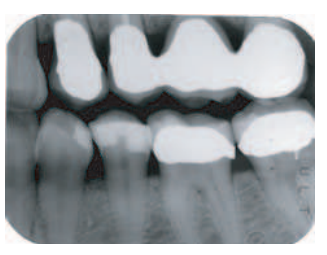

(b)
Figure 1: Postmortem (PM) (a) and antemortem (AM) (b) dental radiographs of the same individual. The "white" region inside the teeth corresponds to dental work.

Our previous work [1], [2] utilized the contours of teeth as cues for identification. Besides tooth contours, the shapes of the dental work (e.g., fillings, crowns and bridges) provide additional information for identification. This paper presents a systematic identification method utilizing both sources of information. A system diagram is given in Fig. 2. The details of each block are presented in the following sections.

\section{Feature Extraction}

The extraction of tooth contours along with some preprocessing procedures, such as radiograph segmentation and gumline detection, has been discussed in the literature [1], [2], [3]. Another useful dental feature is the contours of the dental work. The dental work appears as bright regions in the radiographs (Fig. 1). The contour extraction of dental work is based on fitting a mixture of Gaussians to the intensity histograms - the Gaussian component with the largest mean corresponds to the pixel intensities associated with the dental work. The number of components in the mixture can be estimated using the algorithm proposed by Figueiredo and Jain [4]. The dental work can be segmented using a threshold which best splits the Gaussian component with the largest mean from the other components (See Fig. 3) [5], [6]. 


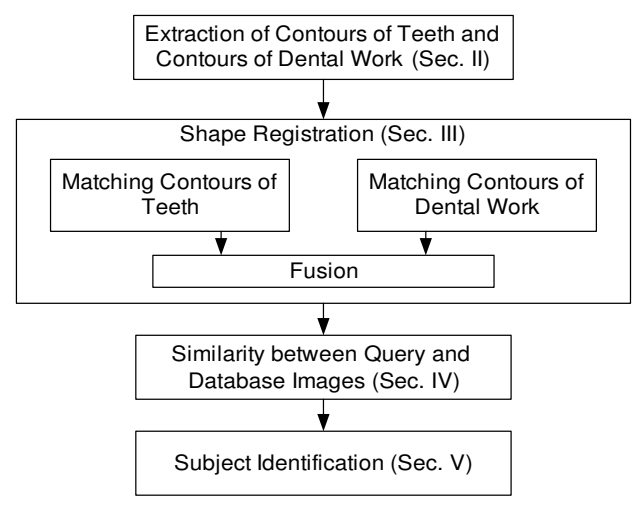

Figure 2: Diagram of the proposed system.

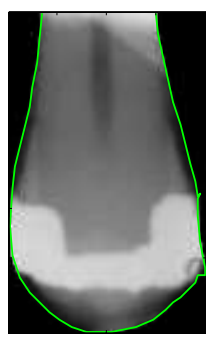

(a)

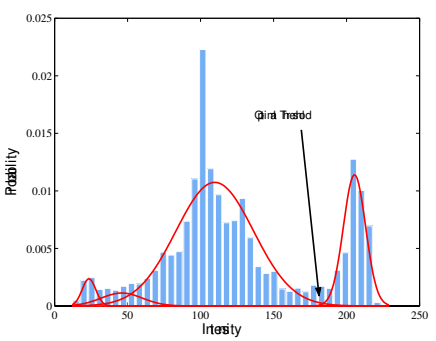

(b)
Figure 3: Fitting a mixture of Gaussians (with 4 components) to the intensity histogram (b) of an image (a).

Intuitively, as the variances of the Gaussians in the mixture decrease (without changing the means), the misclassification error (dental work pixels vs. tooth pixels) decreases as well, because there will be less overlap between the Gaussians. If viewed in the image domain, reduction of variance is equivalent to smoothing the pixels inside each region while preserving the boundary between the regions. We use anisotropic diffusion [7], [8], [9], [10] for this purpose. Fig. 4 shows some smoothed images and the resulting contours, compared with the original images.

\section{Shape Registration}

We first present an algorithm to match the contours of teeth, followed by an algorithm to match the contours of dental work. These two matching scores are then combined using the likelihood estimates.

\subsection{Matching Tooth Contours}

Shape matching problems are usually converted into point matching problems. One approach is to minimize an energy function that involves the amount of transformation and the

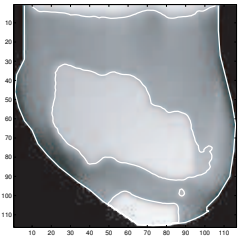

(a)

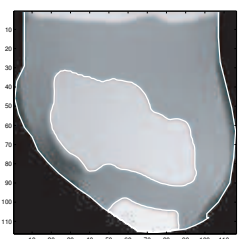

(d)

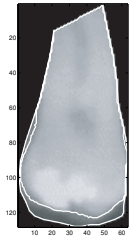

(b)

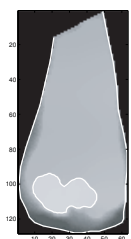

(e)

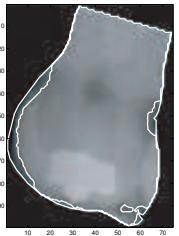

(c)

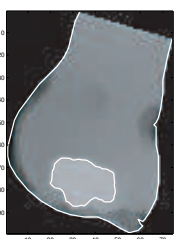

(f)
Figure 4: The original images and the extracted contours ((a), (b), (c)). The corresponding smoothed images and the extracted contours are shown in (d), (e), and (f).

degree of match, such as the softassign algorithm [11]. Another approach is to find the correspondence and to minimize the distance sequentially and iteratively. Our algorithm belongs to the second category. The matching algorithm is as follows:

Algorithm: Matching of Tooth Contours
Input: two shape instances $A$ and $B$ in the form of
spline curves.
1. Polygonal approximation: Approximate continuous
shapes $A$ and $B$ with point sets $A^{\prime}$ and $B^{\prime}$. In order to
find the correspondence from $A^{\prime}$ to $B^{\prime}$, the sampling
rate for $B$ should be sufficiently high so that $B^{\prime}$ can
approximate $B$ precisely enough for distance compu-
tation.
2. Shape registration: If the applied transformation
from $A^{\prime}$ to $B^{\prime}$ is $T$, then find the correspondence from
$T\left(A^{\prime}\right)$ to $B^{\prime}$, which preserves the topologies on the
two curves.
3. Distance computation: The distance between $T\left(A^{\prime}\right)$
and $B^{\prime}$ is the summed distance between all corre-
sponding points. Denote the distance as $d\left(T\left(A^{\prime}\right), B^{\prime}\right)$.
4. Optimization: optimize $d\left(T\left(A^{\prime}\right), B^{\prime}\right)$ in the space
of transformations $\mathbb{R}(T)$.

The details of each step are as follows:

\subsubsection{Polygonal Approximation}

The polygonal approximation of the tooth contours converts the continuous shapes into point sets. We use the arc length parametrization to generate equally spaced points [12]. This prevents the distance between two curves from being biased by some portions of the curve that are densely sampled. 


\subsubsection{Transformation}

Let the rotation be $\theta$, the translations along $x$ and $y$ axes be $t_{x}$ and $t_{y}$, respectively, and the scaling be $s$. A point $(x, y)^{t}$ is mapped to $T(x, y)$ after transformation, where

$$
T(x, y)=s\left(\begin{array}{cc}
\cos \theta & -\sin \theta \\
\sin \theta & \cos \theta
\end{array}\right)\left(\begin{array}{l}
x \\
y
\end{array}\right)+\left(\begin{array}{c}
t_{x} \\
t_{y}
\end{array}\right)
$$

The optimal transformation $T$ minimizes the matching distance. To initialize $T$, both template and target shapes should be normalized so that the widths of their bounding boxes are scaled to the same value.

\subsubsection{Shape Registration}

Shape Registration establishes the correspondence of points between two curves. The registration should be monotonic, i.e., preserve the topologies on the two shapes. In other words, if $a_{i}$ and $a_{j}$ correspond to $b_{k}$ and $b_{l}$, respectively, and $i<j$, then $k<l$ [13]. Because the two curves may not fully overlap, we need to handle the outliers, which do not have corresponding points in the counterpart curve. The registration algorithm is summarized as follows:

\begin{tabular}{|c|}
\hline 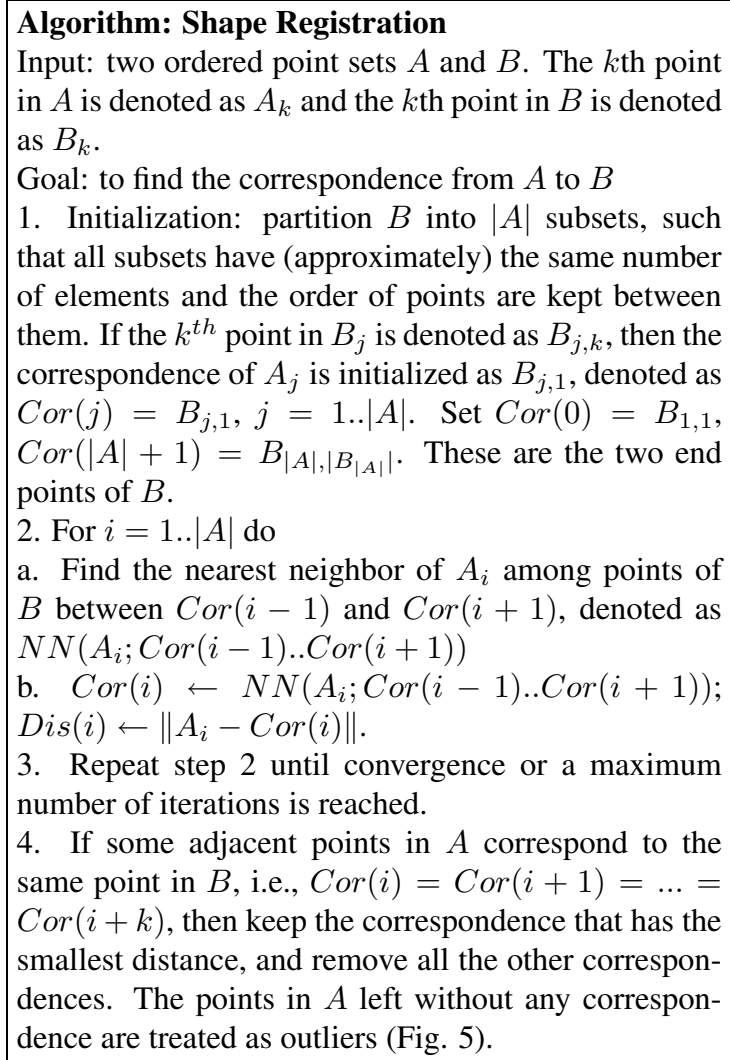 \\
\hline
\end{tabular}

\subsubsection{Distance Computation}

To find the correct transformation and alignment, the distance between the two curves needs to be minimized. Given a correspondence, the distance should not only measure the distance between corresponding points, but also reflect the coverage of the corresponding points in the whole curve.

Given two point sets $A$ and $B$, the correspondence is a bijection between $A^{\prime}$ and $B^{\prime}$, where $A^{\prime} \subseteq A$ and $B^{\prime} \subseteq B$, due to the existence of outliers. The distance between $A^{\prime}$ and $B^{\prime}$ is denoted as $d_{c}\left(A^{\prime}, B^{\prime}\right)$, where

$$
d_{c}\left(A^{\prime}, B^{\prime}\right)=\frac{1}{\left|A^{\prime}\right|} \sum_{\text {all }}\left\|a^{\prime} \in A^{\prime}-\operatorname{Cor}\left(a^{\prime}\right)\right\| .
$$

The distance between $A$ and $B$ is denoted as $d_{t}(A, B)$, where

$$
d_{t}(A, B)=d_{c}\left(A^{\prime}, B^{\prime}\right)+e^{\frac{|A|}{\left|A^{\prime}\right|}} .
$$

The second term in $d_{t}(A, B)$ penalizes the unmatched part of curve $A$. If only a small portion of curve $A$ is matched, the combined matching distance $d_{t}(A, B)$ will be large.

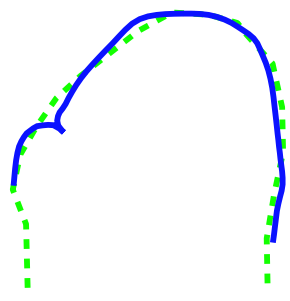

(a)

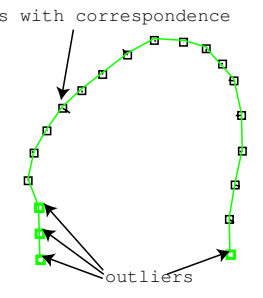

(b)
Figure 5: Shape registration. (a) Aligned contours after optimum transformation; (b) Correspondence after alignment.

\subsection{Matching Contours of Dental Work}

The contour of dental work is another feature for matching dental radiographs. Because the dental work is not always convex, even a slight change in the imaging angle causes a lot of variation in the contours of the dental work (Figs. 6 (a), (b), (c)). To solve this problem, a metric based on the area of the dental work is used.

The preprocessing stage sets the pixels inside the tooth contours to be 0 , and the pixels inside the contours of the dental work to be 1 (See Figs. 6 (d) and (e)). Given two images $M$ and $N$, the number of misaligned pixels $\left(n_{m p}\right)$ between them is defined as:

$$
n_{m p}(M, N)=\frac{\sum_{i, j} M^{\prime}(i, j) \bigoplus N^{\prime}(i, j)}{\sum_{i, j} M^{\prime}(i, j)+N^{\prime}(i, j)},
$$

where $M^{\prime}$ and $N^{\prime}$ are results of the preprocessing on $M$ and $N$, and $\bigoplus$ is the 'exclusive-or' operator. The metric 
$n_{m p}$ is used to measure the alignment of the dental work. If the dental works in images $M$ and $N$ have similar shapes and are well aligned, then $n_{m p}$ is small. So, the distance between the dental work in images $M$ and $N$ is defined as

$$
d_{d w}(M, N)=\min _{\text {for all } T} n_{m p}(T(M), N),
$$

where $T$ is the transformation defined in Equation (1).

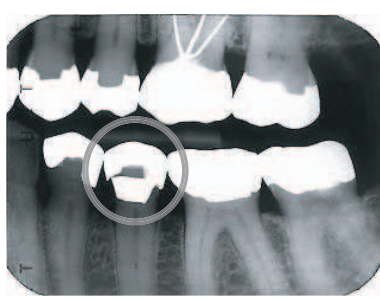

(a)

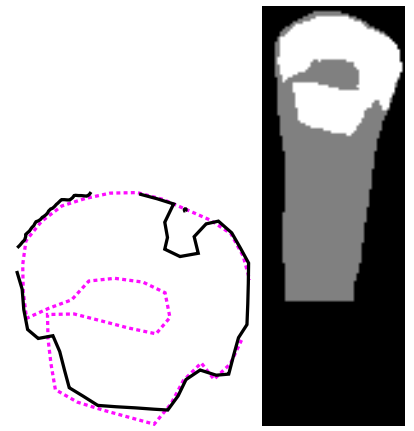

(c)

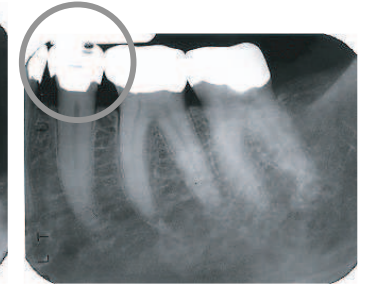

(b)

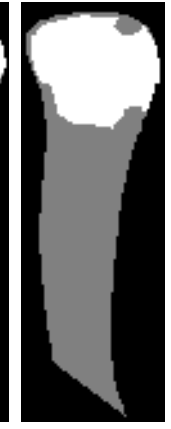

(e)

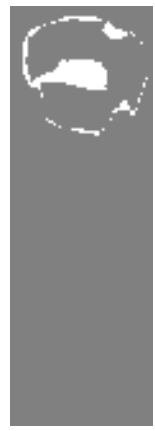

(f)
Figure 6: Dental work merging due to a change in the imaging angle. The hole in the circled dental work seen in image (a) is occluded when viewed from the angle shown in (b) even though both the images are of the same tooth. (c) The contours of the dental work shown in (a) and (b) are aligned. (d) The preprocessed image for the tooth in image (a). (e) The preprocessed image for the tooth in image (b). (f) The result of 'exclusive-or' for the two teeth.

\subsection{Fusion of $d_{t}$ and $d_{d w}$}

Note that the distance $d_{t}$ measures the difference between contours of teeth, and $d_{d w}$ computes the difference between contours of the dental work. We combine these two matching distances to obtain a better similarity measure. However, for teeth without any dental work, $d_{d w}$ is not available. To solve this problem, we propose to combine the distances via likelihood estimates.
Given the distance $d_{d w}$, the two likelihood estimates are

$$
\begin{aligned}
P\left(\omega_{i} \mid d_{d w}\right) & =\frac{p\left(d_{d w} \mid \omega_{i}\right) P\left(\omega_{i}\right)}{p\left(d_{d w}\right)}, \\
P\left(\omega_{g} \mid d_{d w}\right) & =\frac{p\left(d_{d w} \mid \omega_{g}\right) P\left(\omega_{g}\right)}{p\left(d_{d w}\right)},
\end{aligned}
$$

where $\omega_{g}$ represents genuine teeth, and $\omega_{i}$ represents imposter teeth. The distributions $p\left(d_{d w} \mid \omega_{i}\right)$ and $p\left(d_{d w} \mid \omega_{g}\right)$ are obtained through training.

The matching distance $d_{t}$ is combined with $d_{d w}$ to generate the distance $d_{f}$ between the two teeth $A$ and $B$ as follows

$$
\begin{aligned}
& d_{f}(A, B)=d_{t}(A, B) \cdot\left(1+\alpha \cdot\left(T\left(d_{d w}\right)\right)\right), \\
& T\left(d_{d w}\right) \\
& = \begin{cases}P\left(\omega_{i} \mid d_{d w}\right)-P\left(\omega_{g} \mid d_{d w}\right), & \text { if } d_{d w} \text { is available } \\
0, & \text { otherwise }\end{cases}
\end{aligned}
$$

where $\alpha$ is the weight assigned to dental work shapes. The above combination scheme implies that if $d_{d w}$ is more likely to be the distance between the genuine teeth, $d_{t}$ is reduced; if $d_{d w}$ is more likely to be the distance between imposter teeth, $d_{t}$ is amplified; if $d_{d w}$ is not available, $d_{t}$ is not changed.

In practice, the neighboring teeth are matched as a unit, which is denoted as $d_{f}\left(\left[R_{i}, R_{i+1}\right],\left[S_{i}, S_{i+1}\right]\right)$, where $R_{i}$ and $R_{i+1}$ are the neighboring teeth in image $R$, and $S_{i}$ and $S_{i+1}$ are the neighboring teeth in image $S$; the notation $\left[R_{i}, R_{i+1}\right]$ means the concatenation of $R_{i}$ and $R_{i+1}$, and similarly for $\left[S_{i}, S_{i+1}\right]$. This scheme has the advantage that not only the teeth contours but also their relative positions contribute to the matching.

\section{Similarity Between Dental Images}

We view each row of teeth as a separate image. The matching distance between two given images should rely only on the corresponding teeth. Obtaining tooth correspondence between images is the main task at this stage. Exhaustive matching with all possible correspondences is not only time-consuming, but also infeasible, because as the number of teeth in the group increases, the assumption about the rigid transformation (Equation (1)) between images is no longer valid. We assume that no tooth is missing between the acquisitions of AM and PM images, so for neighboring teeth in AM images, their corresponding teeth in PM images should be neighbors as well.

The following algorithm merges the results of shape registration of individual teeth to obtain the correspondence between two images $R$ and $S$. The numbers of teeth in $R$ and $S$ need not to be same. Suppose 
a particular tooth correspondence is denoted as $\mathbb{C}=$ $\left\{\left(R_{i}, S_{j}\right),\left(R_{i+1}, S_{j+1}\right), \ldots,\left(R_{i+l}, S_{j+l}\right)\right\}$, where $R_{i+k}$ is the corresponding tooth of $S_{i+k}, k=0, \ldots, l$. Then the correct tooth correspondence should minimize the average distance between corresponding teeth, which is defined as:

$$
\begin{aligned}
& d_{i m g}(R, S)= \\
& \min _{\text {for all } \mathbb{C}} \frac{1}{l} \sum_{k=0 . . l-1} d_{f}\left(\left[R_{i+k}, R_{i+k+1}\right],\left[S_{i+k}, S_{i+k+1}\right]\right),
\end{aligned}
$$

where $[.,$.$] means the concatenation of neighboring con-$ tours, as mentioned in section 3.3. Fig. 7 shows some examples of tooth correspondences. The distances obtained in this step are used to retrieve AM images from the database.

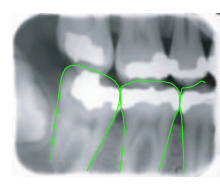

(a)

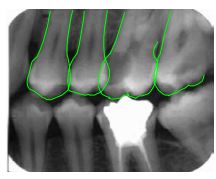

(d)

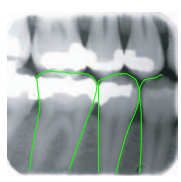

(b)

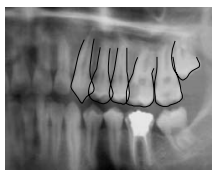

(e)

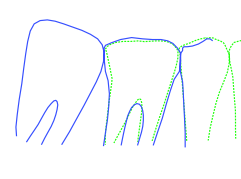

(c)

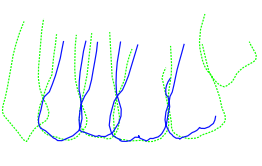

(f)
Figure 7: Two examples of tooth correspondence. The tooth contours in (a) and (b) are paired up in (c); The tooth contours in (d) and (e) are paired up in (f).

\section{Subject Identification}

Given the matching distances between two images, we can now compute the similarity between subjects. Suppose $U$ is a subject to be identified, and denote the PM images for $U$ as $u_{1}, u_{2}, \ldots, u_{m}$. Label the $i$ th subject in the AM database as $V^{i}, i=1, \ldots, k$. Denote the AM images for subject $V^{i}$ as $v_{1}^{i}, v_{2}^{i}, \ldots, v_{n}^{i}$. The algorithm for subject identification has two steps. The first step is to compute the matching distances between one PM image $u_{p}$ and all the AM images of $V^{i}$. If two images do not have any tooth in common, their matching distance will be large. Only if the images have some teeth in common and the correspondence is correct, the matching distance will be small. So the smallest matching distance is chosen to represent the matching distance between the image $u_{p}$ and the subject $V^{i}$. This is denoted as:

$$
d_{i m g_{\_} s u b}\left(u_{p}, V^{i}\right)=\min _{\text {for all } q} d_{i m g}\left(u_{p}, v_{q}^{i}\right) .
$$

In the second step, the image-to-subject distances are averaged over all images of subject $U$ to obtain the matching distance between $U$ and $V^{i}$, that is

$$
d\left(U, V^{i}\right)=\frac{1}{m} \sum_{p=1 . . m} d_{i m g_{-} s u b}\left(u_{p}, V^{i}\right) .
$$

Given the distances between the unidentified subject and all the subjects in the AM database, a ranking of these similarities generates a list of candidates.

\section{Experimental Results}

To evaluate our algorithms, we need a database containing both AM and PM images. PM dental radiographs are much more difficult to be obtained than AM radiographs. We have access to a small database from the FBI's Criminal Justice Information Service (CJIS) division, which is interested in utilizing dental characteristics for identifying Missing and Unidentified Persons (MUP).

In our database there are 11 subjects, each with a number of AM and PM images. There are a total of 158 PM and 156 AM dental radiographs. These images contain 409 PM teeth and 493 AM teeth in total. In our experiment, 5 out of the 11 individuals are randomly selected as training samples and the remaining 6 individuals are used for testing. The process is repeated 25 times for cross-validation.

The dental radiographs are matched in three steps. The first step is shape registration - the PM teeth are matched with the AM teeth individually. To estimate the probability distribution of the matching distances for contours of dental work $\left(p\left(d_{d w} \mid \omega_{i}\right)\right)$ and $\left(p\left(d_{d w} \mid \omega_{g}\right)\right)$, we use a Parzen window approach with Gaussian kernels. By comparing the accuracies of matching teeth (all with dental work) with different values for $\alpha$, the optimum value of $\alpha(\alpha=1)$ is selected. The result of matching against the whole database with $\alpha=1$ shows that in $90 \%$ of the cases, genuine teeth were among the top $15 \%$ of the retrieved images.

In the second step, the teeth are viewed as a group as long as they are in the same row and are neighbors. If a group of PM teeth are matched with the same person's AM teeth and the tooth correspondence is correct, we call it a hit. Some examples of successful and unsuccessful matchings are shown in Fig. 8. The result shows that in $90 \%$ of the cases, the genuine image was among the top $10 \%$ of the retrievals, compared with the top $15 \%$ retrievals after shape registration. Note that the 493 AM teeth are divided into 156 groups and the matching is in terms of groups, so this step has reduced the size of the candidate list, resulting in a higher precision in retrieval.

The purpose of matching dental radiographs is to obtain an individual's identity, so the final stage is to identify the subjects. In this experiment, $6 \mathrm{PM}$ subjects are matched to 


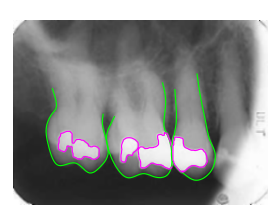

(a)

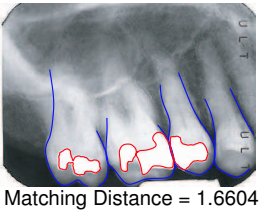

(b)

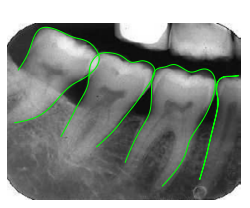

(c)

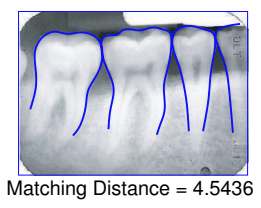

(d)

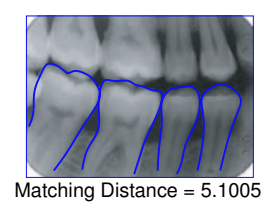

(e)
Figure 8: A successful matching ((a),(b)) and an unsuccessful matching ((c),(d),(e)). Due to the resemblance of the shapes, (c) is incorrectly matched to (d) which belongs to another subject. The genuine match for (c), shown in (e), is ranked as the second closest match.

the 11 AM subjects. For the top-1 retrieval, the upper quartile accuracy among all cross-validation trials is $83 \%$, the lower quartile accuracy is $79 \%$ and the median accuracy is $83 \%$. The median accuracy increases to $100 \%$ if we include the top 2 retrievals.

\section{Conclusions}

Dental biometrics is used to identify individuals in the forensic domain. This paper presents an automatic method for matching dental radiographs. The matchings are performed in three steps. In the first step, a shape registration method is proposed to align and compute the distance between two teeth on the basis of tooth contours. If the shapes of the dental work are available, they can assist in the matching. We propose an area-based metric for matching the dental work. The two matching distances are then combined to obtain the tooth correspondence and to measure the similarity between images. Possible matches for the PM images are identified from a database of AM images. The labels of the retrieved images are used to establish the identity of the deceased subject. Experimental results show that our approach is promising.

There are still a number of challenges that we need to overcome. The shape extraction is a difficult problem for dental radiographs, especially for poor quality images where some tooth contours are indiscernible. For the subjects with a number of missing teeth, we are exploring other features, such as the shape of mandibular canals and maxillary sinus, for subject identification. We are also in the process of obtaining a larger database for the algorithm evaluation.

\section{References}

[1] A. K. Jain and H. Chen, "Matching of dental x-ray images for human identification," Pattern Recognition, vol. 37, no. 7, pp. 1519-1532, 2004.

[2] H. Chen and A. K. Jain, "Tooth contour extraction for matching dental radiographs," proc. 17th ICPR, vol. III, pp. 522525, Cambridge, UK, August, 2004.

[3] J. Zhou and M. Abdel-Mottaleb, "Automatic human identification based on dental x-ray images," in Proc. SPIE Technologies for Homeland Security and Law Enforcement conference, the Biometric Technology for Human Identification conference, Orlando, FL, April 2004.

[4] M. Figueiredo and A. K. Jain, "Unsupervised learning of finite mixture models," IEEE Trans. Pattern Analysis and Machine Intelligence, vol. 24, no. 3, pp. 381-396, 2002.

[5] R. C. Gonzalez and P. Wintz, Digital Image Processing. Addison-Wesley Publishing Company, Inc., 1977.

[6] M. Sonka, V. Hlavac, and R. Boyle, Image Processing Analysis, and Machine Vision, 2nd ed. Florence, KY: Brooks/Cole, Thomson Learning, 1999.

[7] P. Perona and J. Malik, "Scale-space and edge detection using anisotropic diffusion," IEEE Trans. Pattern Analysis and Machine Intelligence, vol. 12, no. 7, pp. 629-639, 1990.

[8] H. Huang and J. Wang, "Anisotropic diffusion for object segmentation," in Proc. IEEE International Conference on Systems, Man, and Cybernetics, vol. 3, Nashville, TN, October 2000, pp. 1563-1567.

[9] Y. Yu and S. Acton, "Speckle reducing anisotropic diffusion," IEEE Trans. Image Processing, vol. 11, no. 11, pp. 1260-1270, 2002.

[10] M. Black, G. Sapiro, D. Marimont, and D. Heeger, "Robust anisotropic diffusion," IEEE Trans. Image Processing, vol. 7, no. 3, pp. 421-432, 1998.

[11] S. Gold, A. Rangarajan, C. Lu, S. Pappu, and E. Mjolsness, "New algorithms for $2 \mathrm{~d}$ and $3 \mathrm{~d}$ point matching: Pose estimation and correspondence," Pattern Recognition, vol. 31, no. 8, pp. 1019-1031, 1998.

[12] H. Wang, J. Kearney, and K. Atkinson, "Arc-length parameterized spline curves for real-time simulation," in Proc. 5th International Conference on Curves and Surfaces, San Malo, France, June 2002, pp. 387-396.

[13] N. Duta, A. K. Jain, and M.-P. Dubuisson-Jolly, "Automatic construciton of $2 \mathrm{~d}$ shape models," IEEE Trans. Pattern Analysis and Machine Intelligence, vol. 23, no. 5, pp. 433-446, May 2001. 